\title{
LOCALIZING EQUIVARIANT BORDISM
}

\author{
E. R. WHEELER
}

\begin{abstract}
The unitary bordism of a finite group is computed up to torsion and an equivariant Rohlin exact sequence is exhibited for groups of odd order.
\end{abstract}

1. Introduction. In [4] Erich Ossa shows that some questions in equivariant bordism can be answered easily when one tensors with the appropriate subring of the rationals. The purpose of this note is to use this technique to understand several facts about equivariant bordism theories. Let $\Omega_{*}^{O}(G), \Omega_{*}^{S O}(G)$, and $\Omega_{*}^{U}(G)$ denote respectively the unoriented, oriented, and unitary bordism of a finite group $G$. Among the results recorded in this paper are:

Proposition 1. If the order of $G$ is $d$, then $\Omega_{*}^{U}(G) \otimes R_{d}$ is a free $\Omega_{*}^{U} \otimes R_{d}$ module on even dimensional generators (where $R_{d}=\{a|b| a$ is any integer and $b$ is a power of $d\}$ ).

Proposition 3. $\Omega_{*}^{U}(G) \otimes_{\Omega_{*}} \Omega_{*}^{o} \rightarrow \Omega_{*}^{O}(G)$ is epic if the order of $G$ (denoted $|G|$ ) is odd.

Proposition 5. $\Omega_{*}^{S O}(G) \otimes_{\Omega_{*} s o} \Omega_{*}^{O} \rightarrow \Omega_{*}^{O}(G)$ is an isomorphism if $|G|$ is odd.

Proposition 7. If $|G|$ is odd, there is an equivariant Dold exact triangle

$$
\Omega_{*}^{S O}(G) \underset{\leftarrow}{\oplus} \Omega_{*}^{O}(G) \stackrel{(2,0)}{\longrightarrow} \Omega_{*}^{S O}(G) \stackrel{\rho}{\longrightarrow} \Omega_{*}^{O}(G) .
$$

2. Preliminaries. It is necessary to consider bordism of 4-tuples $[M, \partial M, \phi, f]$ where

(i) $M$ and $\partial M$ are compact differentiable manifolds where $\partial M$ is the boundary of $M$.

(ii) $\phi: G \times M \rightarrow M$ is a differentiable $G$ action.

(iii) The isotropy subgroups of points in $M$ and $\partial M$ are respectively elements of the families $\mathscr{F}$ and $\mathscr{F}^{\prime}$ of subgroups of $G$.

(iv) $f: M \rightarrow X$ is an equivariant map into the $G$ space $X$.

Received by the editors May 22, 1973 and, in revised form, June 19, 1973.

AMS (MOS) subject classifications (1970). Primary 57D85.

Key words and phrases. Equivariant bordism group, Rohlin exact sequence.

(c) American Mathematical Society 1974 
As in $[7, \S 2]$, the bordism equivalence classes of such objects, $\Omega_{*}^{O}(G)\left(\mathscr{F}, \mathscr{F}^{\prime}\right)(X)$, form an $\Omega_{*}^{O}$ module. If the manifold $M$ has a stable complex structure or an orientation on its tangent bundle which is preserved by the group action, the corresponding bordism theories are denoted respectively by $\Omega_{*}^{U}(G)\left(\mathscr{F}, \mathscr{F}^{\prime}\right)(X)$ and $\Omega_{*}^{S O}(G)\left(\mathscr{F}, \mathscr{F}^{\prime}\right)(X)$ (see Stong [5]). For $L=O, S O$, and $U$ and families of subgroups $\mathscr{F}^{\prime} \subset \mathscr{F}$, there is an exact triangle

$$
\Omega_{*}^{L}(G)\left(\mathscr{F}^{\prime}\right)(X) \stackrel{i_{*}}{\longrightarrow} \Omega_{*}^{L}(G)(\mathscr{F})(X) \stackrel{j_{*}}{\longrightarrow} \Omega_{*}^{L}(G)\left(\mathscr{F}, \mathscr{F}^{\prime}\right)(X)
$$

where $i_{*}$ and $j_{*}$ forget respectively $\mathscr{F}^{\prime}$ and $\mathscr{F}$ freeness and where $\partial$ restricts to the boundary.

One notes that, as in [5, §3], there are universal classifying spaces for real or complex $n$-plane bundles with $G$ action, denoted here by $B O_{n}$ or $B U_{n}$. For $L=O$ or $U$ denote by $F_{K}^{\prime}\left(B L_{n}\right)$ the components of the fixed set of $K$ acting on $B L_{n}$ above which the fibers of the canonical bundle have no trivial irreducible summands when decomposed as $K$-spaces. From Stong's Proposition 5.1 [7] it follows that if $\mathscr{F}^{\prime} \subset \mathscr{F}$ are adjacent families (i.e. $\mathscr{F}-\mathscr{F}^{\prime}=\{K\}$, the elements of the conjugacy class of a subgroup $K$ ), then

$$
\Phi_{K}: \Omega_{*}^{L}(G)\left(\mathscr{F}, \mathscr{F}^{\prime}\right)(X) \rightarrow \bigoplus_{s} \Omega_{*}^{L}\left(N_{G}(K) / K\right)(\{\{1\}\})\left(F_{K}(X) \times F_{K}^{\prime}\left(B L_{s}\right)\right)
$$

is an isomorphism where $\Phi_{K}$ is defined via the fixed set information of $K$. The fixed set of a subgroup $K<G$ acting on $B U_{n}$ is a union of products of $B U_{t}$ 's. If $K$ is a group of odd order, each nontrivial irreducible real representation is the realification of an irreducible complex representation. Hence in this case, $F_{K}^{\prime}\left(B O_{n}\right)$ is a union of products of $B U_{t}^{\prime}$ 's. For an $n$ tuple $\left(t_{1}, \cdots, t_{n}\right)=(t)$, let $B U_{(t)}$ denote the product of $G$-spaces $B U_{t_{i}}$. Then

$$
\Omega_{*}^{L}(G)\left(\mathscr{F}, \mathscr{F}^{\prime}\right)\left(B U_{(t)}\right) \cong \bigoplus \Omega_{*}^{L}\left(N_{G}(K) / G\right)(\{\{1\}\})\left(B U_{(s)}\right)
$$

for all $G$ if $L=U$, and for $G$ of odd order if $L=O$ or $S O$.

Suppose $|G|=d$. The well-known transfer map tells one that, if $\pi: X \rightarrow$ $X / G$ is the quotient map which identifies the $G$ orbits and if $H_{*}\left(X ; R_{d}\right)$ is a free $R_{d}$ module, then $\pi_{*}: \operatorname{INV}\left(H_{*}\left(X ; R_{d}\right)\right) \rightarrow H_{*}\left(X / G ; R_{d}\right)$ is an isomorphism where INV( ) denotes the submodule of elements invariant under the $G$ action [3, Corollary 2.3]. It follows then from the collapse of the bordism spectral sequence for $\Omega_{*}^{U}() \otimes R_{d}$ in this instance that $\Omega_{*}^{U}(X / G) \otimes R_{d} \cong \Omega_{*}^{U} \otimes \operatorname{INV}\left(H_{*}\left(X ; R_{d}\right)\right)$.

3. Unitary equivariant bordism. Note that if $\mathscr{F}$ is the family of all subgroups of $G$, then $\Omega_{*}^{U}(G)(\mathscr{F})(\mathrm{pt})=.\Omega_{*}^{U}(G)$ discussed in the introduction. 
One then has

Proposition 1'. For any family of subgroups of $G, \Omega_{*}^{U}(G)(\mathscr{F})\left(B U_{(t)}\right) \otimes$ $R_{d}$ is a free $\Omega_{*}^{U} \otimes R_{d}$ module on even dimensional generators for any finite group $G$.

Proof. One inducts on the order of $G$ and the size of the family $\mathscr{F}$. If $\mathscr{F}=\{\{1\}\}$, then [7, Proposition 5.2] yields that $\Omega_{*}^{U}(G)(\{\{1\}\})\left(B U_{(t)}\right) \otimes$ $R_{d} \cong \Omega_{*}^{U}\left(B U_{(t)} \times{ }_{G} E G\right) \otimes R_{d} \cong \Omega_{*}^{U} \otimes \operatorname{INV}\left(H_{*}\left(B U_{(t)} ; R_{d}\right)\right)$ where $E G$ is the total space of a universal principal $G$ bundle and $G$ acts diagonally on $B U_{(t)} \times E G$.

To complete the induction step, choose a maximal subgroup $K$ of a family $\mathscr{F}$. Let $\mathscr{F}^{\prime}=\mathscr{F}-\{K\}$. One has the long exact sequence

$$
\begin{array}{r}
\Omega_{*}^{U}(G)\left(\mathscr{F}^{\prime}\right)\left(B U_{(t)}\right) \otimes R_{d} \rightarrow \Omega_{*}^{U}(G)(\mathscr{F})\left(B U_{(t)}\right) \otimes R_{d} \rightarrow \bigoplus \Omega_{*}^{U}\left(N_{G}(K) / K\right) \\
(\{\{1\}\})\left(B U_{(t)}\right) \otimes R_{d}
\end{array}
$$

where the first and third modules are free $\Omega_{*}^{U} \otimes R_{d}$ modules on even dimensional generators. Since $\Omega_{\text {odd }}^{U}=0$, the long sequence is in fact a split short exact sequence and the result follows.

There is the fixed point set map

$$
\Phi_{\mathscr{F}}: \Omega_{*}^{U}(G)(\mathscr{F}) \rightarrow \underset{\{H\} \subset \mathscr{F}(t)}{\bigoplus_{*}} \Omega_{*}^{U}\left(B U_{(t)}\right)
$$

defined by picking a representative of each conjugacy class $\{H\} \subset \mathscr{F}$, considering the fixed set of each representative $H$, decomposing the normal bundle to the fixed set via the nontrivial representations of $H$, and classifying the pieces into the appropriate $B U_{t}$. One sums over the representatives and the components of the fixed sets to complete the definition of $\Phi_{\mathscr{F}}$ $($ see $[7, \S 12])$.

Proposition 2. $\quad \Phi_{\mathscr{F}}: \Omega_{*}^{U}(G)(\mathscr{F}) \otimes R_{d} \rightarrow \bigoplus \Omega_{*}^{U}\left(B U_{(t)}\right) \otimes R_{d}$ is monic for all families.

Proof. If $\mathscr{F}=\{\{1\}\}$, then $\Omega_{*}^{U}(G)(\{\{1\}\})=\tilde{\Omega}_{*}^{U}(B G) \oplus \Omega_{*}^{U}$ and the reduced bordism of $B G$ consists of $d$ torsion. Letting $\mathscr{F}^{\prime} \subset \mathscr{F}$ be adjacent families differing by $\{K\}$, one has:

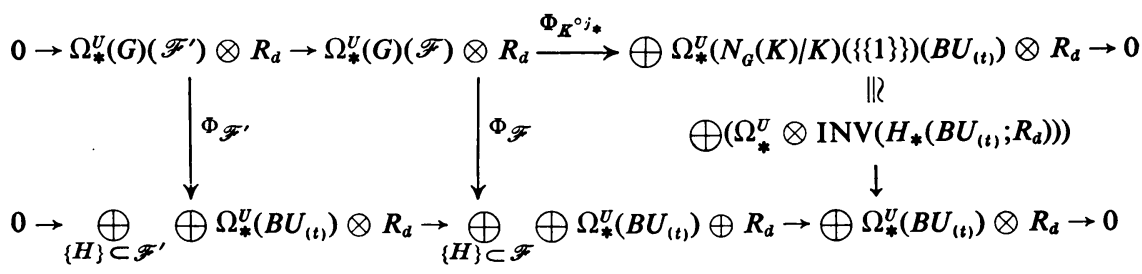


The diagrams commute, the short exact sequences are split exact, the third map is monic and one may assume the first map is monic by induction. Hence the second map is monic.

One notes that for a group of odd order the nontrivial irreducible complex representations have the property that a representation is not equivalent to its conjugate [9, Theorem 4.7.3]. Let $I(K)$ be the nontrivial irreducible complex representations of $K . N_{G}(K) / K$ acts on $I(K)$ with $(g, \pi)=\pi^{g}$ where $\pi^{g}(k)=\pi\left(g^{-1} k g\right)$ for $k \in K$. It follows that $\pi^{g} \neq \bar{\pi}$ for any $g \in N_{G}(K) / K$ so that $I(K)=A \cup A^{\prime}$ where $A$ and $A^{\prime}$ are disjoint $N_{G}(K) / K$ invariant sets with $A^{\prime}=\{\bar{\pi} \mid \pi \in A\}$. Hence the $N_{G}(K) / K$ space $F_{K}^{\prime}\left(B U_{t}\right)$ decomposes into $N_{G}(K) / K$ invariant pieces $F_{K, A}^{\prime}\left(B U_{t}\right) \cup D$ where $D$ consists of those components of $F_{K}^{\prime}\left(B U_{t}\right)$ such that the $K$ decomposition of the fibers of the canonical bundle contains at least one summand from $A^{\prime}$. Now it is clear that the realification map $r$ : $F_{K, A}^{\prime}\left(B U_{t}\right) \rightarrow F_{K}^{\prime}\left(B O_{2 t}\right)$ is a homotopy equivalence and is equivariant. Hence the map induced by $r \times 1$ from

$$
F_{K \cdot A}^{\prime}\left(B U_{t}\right) \underset{N_{G}(K) / K}{\times} E\left(N_{G}(K) / K\right) \rightarrow F_{K}^{\prime}\left(B O_{2 t}\right) \underset{N_{G}(K) / K}{\times} E\left(N_{G}(K) / K\right)
$$

is a homotopy equivalence. Then one has:

Proposition 3'. If $|G|=d$ is odd, $\rho: \Omega_{*}^{U}(G)(\mathscr{F}) \otimes_{\Omega_{*} v} \Omega_{*}^{o} \rightarrow \Omega_{*}^{o}(G)(\mathscr{F})$ is epic for all families $\mathscr{F}$ where $\rho$ is induced by the map which forgets complex structure.

Proof. One inducts as before. For $\mathscr{F}^{\prime} \subset \mathscr{F}$ adjacent families one has:

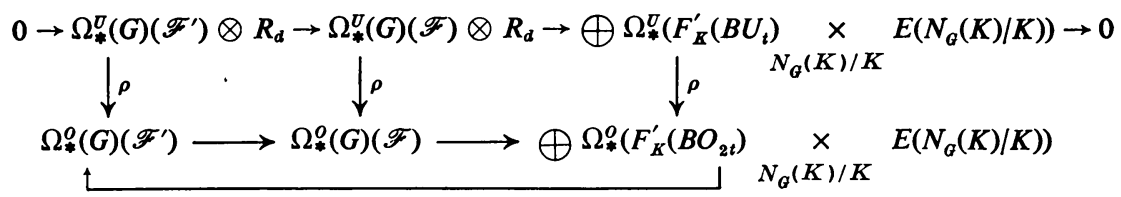

The top sequence is split exact so tensoring with $\Omega_{*}^{O} \otimes R_{d}$ over $\Omega_{*}^{U} \otimes R_{d}$ preserves exactness and yields

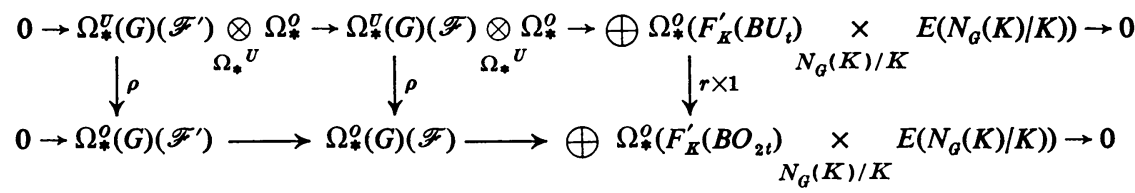

The first and third maps are epic by induction and the comments preceding this proposition, the top sequence is split exact, and this completes the proof. 
Note. Using the same arguments one can show that is epic.

$$
\rho:\left(\Omega_{*}^{U}(G)(\mathscr{F}) \otimes R_{d}\right) \underset{\Omega_{*} \otimes \otimes R_{d}}{\otimes}\left(\Omega_{*}^{S O} \otimes R_{d}\right) \rightarrow \Omega_{*}^{S O}(G)(\mathscr{F}) \otimes R_{d}
$$

\section{Oriented equivariant bordism.}

Proposition 4. If $|G|=d$ is odd, then $\Omega_{*}^{S O}(G)(\mathscr{F}) \otimes R_{d}$ is a free $\Omega_{*}^{S O} \otimes R_{d}$ module on even dimensional generators.

Proof. One follows the arguments of Proposition 1' while noting that the comment following Proposition $3^{\prime}$ shows the exact sequence to be split.

Note. If $G$ is abelian, Propositions 1 and 4 are somewhat weaker than previously known results (see [4] and [8]).

Proposition 5'. If $|G|=d$ is odd then

$$
\Omega_{*}^{S O}(G)(\mathscr{F}) \underset{\Omega_{*}^{s o}}{\otimes} \Omega_{*}^{O} \rightarrow \Omega_{*}^{O}(G)(\mathscr{F})
$$

is an isomorphism for all families $\mathscr{F}$.

Proof. The proof follows as in Proposition 3'.

There is the standard Rohlin exact sequence $\Omega_{*}^{S O} \stackrel{2}{\rightarrow} \Omega_{*}^{S O} \stackrel{\rho}{\rightarrow}_{\rightarrow} \Omega_{*}^{O}$ where $\rho$ forgets the orientation. It was observed by Dold that the Rohlin sequence fits into an exact triangle

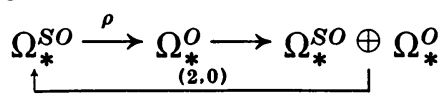

(see [6, p. 216]). Using Proposition 5 one is able to arrive at equivariant versions of these exact sequences.

Proposition 6. If $|G|=d$ is odd, there is an equivariant Rohlin exact sequence,

$$
\Omega_{*}^{S O}(G) \stackrel{2}{\longrightarrow} \Omega_{*}^{S O}(G) \stackrel{\rho}{\longrightarrow} \Omega_{*}^{O}(G) .
$$

Proof. Defining $\sigma: B \rightarrow B \otimes R_{d}$ by $\sigma(b)=b \otimes 1$, one has

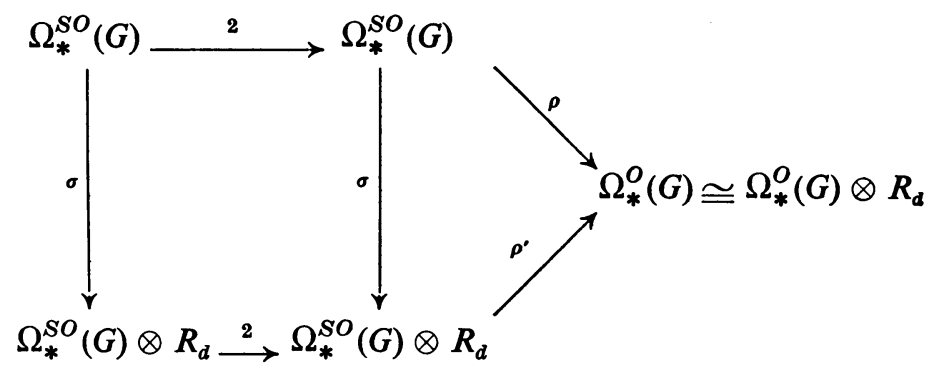


in which the bottom sequence is exact since it results from tensoring the standard Rohlin exact sequence by the free module $\Omega_{*}^{S O}(G) \otimes R_{d}$. If $\rho(x)=0$ then $\rho^{\prime} \sigma(x)=0$ so there is $y^{\prime} \in \Omega_{*}^{S O}(G) \otimes R_{d}$ with $2 y^{\prime}=\sigma(x)$. There is $y \in \Omega_{*}^{S O}(G)$ with $\sigma(y)=d^{t} y^{\prime}$ so $\sigma\left(2 y-d^{t} x\right)=0$. The kernel of $\sigma$ is $d$-torsion so $2 d^{s} y=d^{t+s} x$ or $d^{t+s} x$ is in the image of 2 . Since $d$ is odd, $x$ is in the image of 2 . Clearly $\rho \circ 2=0$.

Note. P.E. Conner has shown $\Omega_{*}^{S O}\left(Z_{2}\right)^{2} \rightarrow \Omega_{*}^{S O}\left(Z_{2}\right) \stackrel{\rho}{\rightarrow} \Omega_{*}^{O}\left(Z_{2}\right)$ is not exact [1, Theorem 5.8].

Proposition 7. If $|G|=d$ is odd, there is an equivariant Dold exact triangle

$$
\Omega_{*}^{S O}(G) \underset{+}{\oplus} \Omega_{*}^{O}(G) \stackrel{(2,0)}{\longrightarrow} \Omega_{*}^{S O}(G) \stackrel{\rho}{\longrightarrow} \Omega_{*}^{O}(G)
$$

induced by tensoring the standard Dold exact triangle with $\Omega_{*}^{S O}(G)$ over $\Omega_{*}^{S O}$.

Proof. One tensors the above triangle with $R_{d}$ (thus insuring exactness) and compares the two via the homomorphism $\sigma: B \rightarrow B \otimes R_{d}$ as in Proposition 6. Exactness at $\Omega_{*}^{S O}(G)$ is the Rohlin exact sequence. Exactness at $\Omega_{*}^{O}(G)$ follows since $\Omega_{*}^{O}(G)$ is a $Z_{2}$ vector space. $(2,0) \circ \alpha=0$ since the image of $\alpha$ is torsion of order 2 . Kernel of $(2,0)$ is the torsion of order 2 and hence by comparison via $\sigma$ is in the image of $\alpha$.

Note. In the Dold exact triangle the map $\Omega_{*}^{O} \rightarrow \Omega_{*}^{S O} \oplus \Omega_{*}^{O}$ is defined by taking the dual submanifolds of the first and second powers of the first Stiefel-Whitney class. In the equivariant situation due to the lack of equivariant transverse regularity the map appears to be purely algebraic.

\section{REFERENCES}

1. P. E. Conner, "Orientation preserving involutions", in Lectures on the action of a finite group, Lecture Notes in Math., no. 73, Springer-Verlag, Berlin and New York, 1968, Chap. 2. MR 41 \#2670.

2. P. E. Connor and E. E. Floyd, Differentiable periodic maps, Ergebnisse der Math. und ihrer Grenzgebiete, Band 33, Springer-Verlag, Berlin; Academic Press, New York, 1964. MR 31 \#750.

3. E. E. Floyd, Periodic maps via Smith theory, Ann. of Math. Studies, no. 46, Chapter III, Princeton Univ. Press, Princeton, N.J., 1960.

4. E. Ossa, Unitary bordism of abelian groups, Proc. Amer. Math. Soc. 33 (1972), 568-571. MR 45 \#2743.

5. R. E. Stong, Complex and oriented equivariant bordism, Topology of Manifolds (Proc. Inst., Univ. of Georgia, Athens, Ga., 1969), Markham, Chicago, Ill., 1970, pp. 291-316. MR 42 \#8521.

6. - Notes on cobordism theory, Mathematical Notes, Princeton Univ. Press, Princeton, N.J.; Univ. of Tokyo Press, Tokyo, 1968. MR 40 \#2108.

7. - Unoriented bordism and actions of finite groups, Mem. Amer. Math. Soc., no. 103 (1970). MR 42 \#8522. 
8. E. R. Wheeler, The oriented bordism of cyclic groups, Dissertation, University of Virginia, Charlottesville, Va., 1973.

9. J. A. Wolf, Spaces of constant curvature, Chapter 4, McGraw-Hill, New York, 1967. MR 36 \#829.

Department of Mathematics, University of Virginia, Charlottesville, VIRGINIA 22901

Current address: Department of Mathematics, Northern Kentucky State College, Highland Heights, Kentucky 41076 